\title{
THE THYROID UNDER THREAT IN A WORLD OF PLASTICS
}

\author{
Leonidas H. Duntas
}

Evgenidion Hospital, Unit of Endocrinology, Metabolism and Diabetes, Thyroid Section, University of Athens, Greece

\begin{abstract}
Among the various categories of thyroid disruptors, plasticizers, particularly phthalates and bisphenol A and substitutes, are most frequently examined due to their very extensive use and extreme durability. Both experimental and clinical studies have shown the deleterious effects of plasticizers on, among other major organ systems, thyroid physiology and thyroid hormone metabolism. Though the mechanism(s) are not as yet well clarified, it is hypothesized that plasticizers exert a suppressive effect on thyroid function and disrupt thyroid signaling. Similar effects have been reported in wildlife, which is also increasingly exposed to the plastic contamination of both solid and aqueous environments. By presenting the results of several recently published large studies linking plastics to thyroid dysfunction, this review aims to sound the alarm and thus call to action all academic organizations in order to counteract a threat which imperils not only the thyroid and the reproductive system but also the entirety of life on our planet.
\end{abstract}

Key words: thyroid, TSH, phthalates, bisphenol-A, endocrine disruptors, hypothyroidism.

\section{Introduction}

Contemporary technological progress has, over the past century, introduced as many as 85,000 new chemicals, without, however, the existence of adequate elimination systems to cope with this onslaught [1]. Thus, with regard to the impact on the human organism, multiple toxins are now entering the body through the air and water, but also via such products as food, makeup and plastic items, incurring an estimated $\$ 359$ billion a year in healthcare costs. The presentday overwhelming accumulation in our environments of toxic disruptors of endocrine systems (endocrine disruptors or EDs), particularly of the thyroid (and, by extension, of reproductive function), has impelled thyroidologists to identify and categorize a wide array of toxins affecting thyroid function for the purpose of pressing for remedial action [2,3]. Toxic interference of thyroid function can occur at several levels, including iodine uptake, thyroid hormone (TH) production and TH metabolism and/or action, and several recent reviews have analytically enumerated these endocrine disruptors and recorded their highly detrimental effects [4-6].

This brief review aims to underscore the degree to which plasticizers, particularly phthalates and bisphenol A, seriously affect thyroid function in humans, as well as in animals, thereby sounding the alarm as to the grave impact of the thousands of plastics that are today flooding all planetary environments.

Correspondence to: Leonidas Duntas, M.D.

Evgenideion Hospital, Unit of Endocrinology, Metabolism and Diabetes, Thyroid Section, University of Athens, 20 Papadiamantopoulou Str., Athens 11520, Greece

Phone: 0030210.674 .8878

E-mail: ledunt@otenet.gr

Received January $12^{\text {th }}, 2018$

\section{Plasticizers}

\section{Phthalates and the thyroid}

Plasticizers are synthetic polymers, most commonly polyvinyl chloride (PVC), added especially to rubbers and resins, to improve the flexibility, transparency and durability of the material. Plasticizers used in PVC and other plastics are usually esters of polycarboxylic acids, these including sebacates, adipates, phthalates and terephthalates, azelates, as well as a number of other blends.

Phthalate esters are commonly used in a variety of household applications such as shower curtains, flooring, food containers, wrappers, cleaning materials and personalcare products, including perfumes, eye shadow, nail polish, liquid soap and hair spray [7], also footwear, sporting goods, toys, numerous baby items, car interiors, medical devices. Because they are not chemically bonded to the host plastics, phthalates, esters of phthalic acid, of which the most widely used are di(2-ethylhexyl) phthalate (DEHP), diisodecyl phthalate (DIDP) and diisononyl phthalate (DINP), can easily be released by heating or by extraction and, when absorbed in the body become EDS. The phthalates DIDP and DINP enter the human body via ingestion, inhalation and dermal absorption, while the main source of DEHP is the diet, and particularly fatty foods, milk, butter and meats. Exposure to DEHP also takes place in hospital environments where DEHP leaches directly into liquids that go through PVC/DEHP tubing and equipment. Interestingly, despite the common belief, exposure to phthalates is greater from ingestion of certain foods than through bottled water [8]. Meanwhile, two studies conducted in the USA and Norway between 2003 and 2010 analyzing data from 9,000 individuals found that those who frequently consumed the highly processed, 
packaged and handled food from fast food restaurants and junk food hangouts had significantly higher levels of two separate phthalates, DEHP and DINP, in their urine samples $[9,10]$. Since these chemicals leach into fast food and junk food from a variety of sources, e.g. packaging, cans, food gloves, this type of eating exposes the public to EDs.

Over the past decade, a number of regulations have been implemented with regard to numerous products containing phthalates, with DEHP having been phased out in Europe by Annex XIV of the European Union's $\mathrm{REACH}$ legislation. Under REACH, DEHP can only be used in specific cases if authorization has been granted. Authorizations are granted by the European Commission after obtaining the opinion of the Committee for Risk Assessment (RAC) and the Committee for Socio-economic Analysis (SEAC) of the European Chemicals Agency (ECHA).

Phthalates, albeit inconsistently, have been implicated in thyroid dysfunction and disease. In a very recent population-based cross-sectional study of 279 Taiwanese adults and 79 minors, urine phthalate metabolites were measured together with serum indicators of thyroid function [12]. Serum T4 levels were negatively associated with urinary mono-phthalate and the sum of urinary DEHP metabolite levels. Free thyroxine (FT4) levels were negatively associated with urinary mono-ethylhexyl phthalate (MEHP) levels but positively associated with urinary monophthalate, indicating that phthalates may impact thyroid function, even though causality was not definitively identified.

In another study with 6,003 participants from the Korean National Environmental Health Survey (KoNEHS), 2012-2014, DEHP metabolites, along with benzyl-butyl phthalate (BBzP) and di-butyl phthalate (DBP) metabolites and BPA were measured in urine [13]. Association studies were also performed with TH and TSH levels quantified in serum. In general, urinary phthalate metabolites were associated with lowered total T4 or T3 or increased TSH levels in serum. When grouped by sex, urinary metabolites of phthalates were inversely associated with $\mathrm{T} 4$ only among males. Among females, mono-benzyl phthalate (MBzP) and mono-n-butyl phthalate (MnBP) levels were inversely associated with TSH and T3, respectively. In addition, a negative association between BPA and TSH was observed.

In China, a recent cross-sectional study was carried out in 216 children aged 5-7 years. Separate collections were made of urinary concentrations of eight monophthalate metabolites (MPAEs) from children from both urban and rural areas and an investigation was conducted into their associations with thyroid function and growth hormones [14]. It was ascertained that children from urban areas had higher MPAE concentrations than those from rural areas, while the majority of MPAEs were positively associated with free triiodothyronine (FT3) and FT4. Moreover, insulin-like growth factor 1 (IGF-1) concentration variably diminished by between $0.082 \mathrm{ng} / \mathrm{mL}$ and $0.132 \mathrm{ng} / \mathrm{mL}$ with each $1 \mathrm{ng} / \mathrm{mL}$ increase in phthalate metabolites, while the concentration of insulin-like growth factor binding protein 3 decreased by $0.01 \mathrm{mg} / \mathrm{L}$ with each $1 \mathrm{ng} / \mathrm{mL}$ increase in MPAE. The latter results point to the possibility that exposure to certain phthalates in childhood could interfere with $\mathrm{TH}$ and growth.

In rodents, the effects of short-term fetal exposure to phthalates on the male reproductive system were unequivocally shown; however, information on the longterm effects of DEHP in utero exposure on gonadal function and other organs are at present sparse [15]

Other animal and wildlife studies have presented novel findings suggesting that in various species DEHP exerts more complex and wider disruptive effects on the endocrine system, including the thyroid and metabolism. For example in fish, numerous environmental stressors exert variable effects, depending on the duration of exposure, on the fish thyroid cascade, these effects possibly mediated via imbalance of plasma T4 and T3 levels or damage to the structure of thyroidal tissues [16]. The thyroidal system in vertebrates is strongly linked to other endocrine systems including the control of reproduction. Therefore, any chemical interference in fish thyroid function due to environmental stressors can potentially have highly damaging effects on several facets of reproduction: this includes inhibition of sperm production as well as decrease of egg production, gonad development, ovarian growth, swimming activity and fertilization and, finally, an increase in larval mortality [16].

\section{Bisphenol A, bisphenol S and the thyroid}

Bisphenol A (BPA), a high volume chemical compound of polycarbonate plastic that is widely used for food storage, is likely to be harmful to both human and animal life, most particularly after contact with hot liquids. BPA, as well as phthalates, can leach, migrate or off-gas from products over time and enter the body where it is rapidly metabolized and excreted in urine, with an elimination half-life of less than $24 \mathrm{hr}$ [17].

BPA is ingested in high amounts from canned foods, in contrast to minimal intake from non-canned foods [18]. The monotonic association observed between fast food meat intake and BPA, in contrast to the generally low (1$3 \mathrm{ng} / \mathrm{g}$ ) levels of BPA in fast food components, although not in hamburgers $(10,9 \mathrm{ng} / \mathrm{g})$, indicate that hamburgers may be a major source of BPA exposure [19].

Despite abundant evidence that BPA is contributing to adverse effects on human health and notwithstanding increasing consumer pressure, to date the FDA, though restricting has not yet banned the use of BPA in baby bottles, a decision thought to be based on industry recommendations [20], while the US Regulatory Commission continues to declare that there is an adequate margin of safety. Indeed, any attempt to remove the substance from the food supply has failed, excepting from baby bottles. This constitutes the epitome of illogicality, since the scientific community strongly believes that BPA is an ED and should therefore, logically, no longer be part of the food chain. Recently, bisphenol S (BPS) was introduced as a substitute for BPA in products claiming to be "BPA free". However, BPS is proving to be no less harmful than BPA 
(Figure 1), having been detected in many everyday items, from canned drinks to receipt papers [20]. Studies have pointed to its negative impact on the reproductive system as well as its tendency to multiply fat cells, thereby causing obesity and diabetes. Its effects are observed to be related to timing and length of exposure.

\section{Bisphenol A}<smiles></smiles>

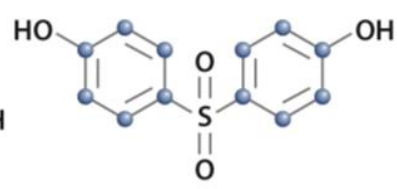

Fig. 1 Chemical structure of Bisphenol A and Bisphenol S

With regard specifically to thyroid signaling, an in vivo and in vitro model (the ZEBRA study) that examined the dose and time effects of BPA on TH synthesis observed altered expression of the genes involved in $\mathrm{TH}$ synthesis and of thyroid specific transcriptional factors [21]. In other words, it is likely that BPA exerts direct effects on thyroid follicular cells, albeit the mechanisms, which are complex, are not as yet well clarified [22]. In this line of evidence, it has recently been reported, through the conduct of a series of in vitro and in vivo assays, that BPA, as well as bisphenol $\mathrm{F}$ (BPF), another alternative to BPA, interferes with the TH signaling pathway, presumably via a number of different pathways [23]. In the fluorescence competitive binding assay, both BPA and BPF were bound to TH receptors (TR $\alpha$ and TR $\beta$ ), with an order of magnitude lower than BPA. Furthermore, a BPA substitute, BPS, affects TH homeostasis and $\mathrm{TH}$ synthesis at lower doses than does BPA, which could render the substitute more hazardous than BPA [24].

In a cross-sectional study from Thailand, the Thai National Health Examination Survey IV 2009, the relationship between BPA exposure and thyroid function was studied in 2,340 subjects aged 18-94 years [25]. BPA was detected in $52.8 \%$ of serum samples; a significantly negative correlation was found between serum BPA and FT4 levels in males only, while no association was registered with TSH in either gender [25]. In the authors' view, this gender-related association may be linked to androgendependent differences in the metabolism of BPA.

In another cross-sectional study from the NHANES 2007-2008 survey, the relationship between urinary phthalate and BPA with serum TH and TSH concentrations was analyzed [26]. While inverse relationships were noted in adults between urinary BPA and total T4 and TSH, significant positive relationships were observed between phthalate metabolites and total T3 among adolescents. Although these study results are not suggestive of any causal relationship a thyroid disrupting effect of BPA can be postulated. This effect may be directly suppressive on thyroid follicular cells and could also be gender- and androgen-dependent.

Animal studies have provided data demonstrating that BPA disrupts TH during pregnancy [27]. Meanwhile, a very up-to-date study in 116 cases of preterm birth and 323 controls showed for the first time in humans that BPA and TSH were inversely associated and that an interquartile range increase in BPA was associated with an $8.21 \%$ decrease in TSH: this relation was repeatedly confirmed during the follow-up visits. The issue is a very serious one, since the latter consistent inverse association of BPA with TSH during pregnancy could well affect birth outcome and child development [28].

Another study from China revealed that there is a link between high BPA levels and excessive iodine intake in patients with papillary thyroid carcinoma and nodular goiter (NG) [29]. Though there were no sexspecific differences for total BPA levels measured in serum and in urine and urinary iodine concentrations (UIC), a significant correlation between BPA and UIC was found in those with PTC and NG. The results possibly point to an association between the metabolic pathways of BPA and iodine in the pathogenesis of PTC and NG.

\section{Conclusions}

This review of some of the very latest data on plastic contamination worldwide provides unambiguous evidence that the plastic additives, phthalates as well as BPA and its substitutes, are present in a very wide range of consumer products, this resulting in considerable exposure of populations everywhere to these very toxic substances. Studies in humans and in animals have substantiated associations between exposure to phthalates and BPA and serum TH levels, but confirmation on a larger scale is essential. In the meantime, it is incumbent upon the medical community to seriously consider the deleterious effects of BPA (and substitutes) on all body systems and on metabolism and to press for change. On the basis of the steadily increasing evidence of the disruption of thyroid function due to these currently omnipresent toxic chemicals, it is evident that, at the very least concerning all food products, a total ban on BPA and its substitutes must be swiftly implemented.

\section{References}

1. Fourth National Report on Human Exposure to Environmental Chemicals. U.S. CDC 2009

2. Brent GA. Environmental Exposures and Autoimmune Thyroid Disease. Thyroid 2010; 20:755-761

3. Pearce EN, Braverman LE 2009 Environmental pollutants and the thyroid. Best Pract Res Clin Endocrinol Metab 2009; 23:801-813.

4. Brucker-Davis F. Effects of environmental synthetic chemicals on thyroid function. Thyroid 1998; 8:827-856.

5. Zoeller TR. Environmental chemicals targeting thyroid. Hormones 2010; 9:28-40.

6. Duntas LH. Chemical contamination and the thyroid. Endocrine 2015; 48:53-64. doi: 10.1007/s12020-014-0442-4 
7. Lorz PM, Towae FK, Enke W, Jäckh R, Bhargava N, Hillesheim W. Phthalic acid and derivatives. In Ullmann's Encyclopedia of Industrial Chemistry, 2007, Wiley-VCH, Weinheim. doi:10.1002 /14356007

8. Erythropel, HC, Maric M, Nicell J, Leask RL, Yargeau V. Leaching of the plasticizer di(2-ethylhexyl)phthalate (DEHP) from plastic containers and the question of human exposure. Appl Microbiol Biotechnol 2014; 98:9967-9981. doi:10.1007/s00253014-6183-8

9. Zota, Ami R.; Phillips, Cassandra A.; Mitro, Susanna D. Recent fast food consumption and bisphenol A and phthalates exposures among the U.S. population in NHANES, 2003-2010. Environmental Health Perspectives 2016; 124:1521-1528. doi:10.1289/ehp. 1510803

10. Sakhi AK, Lillegaard ITL, Voorspoels S, Carlsen MH, Løken EB, Brantsæter AL, et al. Concentrations of phthalates and bisphenol A in Norwegian foods and beverages and estimated dietary exposure in adults. Environ Int 2014; 73:259-269.

11. https://echa.europa.eu/information-on-chemicals/cl-inventorydatabase/-/discli/details/10536

12. Huang HB, Pan WH, Chang JW, Chiang HC, Guo YL, Jaakkola JJ, Huang PC. Does exposure to phthalates influence thyroid function and growth hormone homeostasis? The Taiwan Environmental Survey for Toxicants (TEST) 2013. Environ Res 2017; 153:63-72. doi: 10.1016/j.envres.2016.11.014.

13. Park C, Choi W, Hwang M, Lee Y, Kim S, Yu S, Lee I, Paek D, Choi K. Associations between urinary phthalate metabolites and bisphenol A levels, and serum thyroid hormones among the Korean adult population - Korean National Environmental Health Survey (KoNEHS) 2012-2014. Sci Total Environ 2017; 584-585:950-957. doi: 10.1016/j.scitotenv.2017.01.144

14. Wu W, Zhou F, Wang Y, Ning Y, Yang JY, Zhou YK Exposure to phthalates in children aged 5-7years: Associations with thyroid function and insulin-like growth factors Sci Total Environ. 2017 Feb 1;579:950-956. doi: 10.1016/j.scitotenv.2016.06.146.

15. Martinez-Arguelles DB1, Campioli E, Culty M, Zirkin BR, Papadopoulos V. Fetal origin of endocrine dysfunction in the adult: the phthalate model. J Steroid Biochem Mol Biol 2013; 37:5-17. doi: 10.1016/j.jsbmb.2013.01.007.

16. Nugegoda D, Kibria G. Effects of environmental chemicals on fish thyroid function: Implications for fisheries and aquaculture in Australia. Gen Comp Endocrinol 20171; 244:40-53. doi: 10.1016/j.ygcen.2016.02.021.

17. Johns LE, Cooper GS, Galizia A, Meeker JD. Exposure assessment issues in epidemiology studies of phthalates. Environ Int 2015; 85:27-39

18. Geens T, Aerts D, Berthot C, Bourguignon JP, Goeyens L, Lecomte $\mathrm{P}$, et al. A review of dietary and non-dietary exposure to bisphenolA. Food Chem Toxicol 2012; 50:3725-3740.
19. Cao XL, Perez-Locas C, Dufresne G, Clement G, Popovic S, Beraldin F, et al. Concentrations of bisphenol $\mathrm{A}$ in the composite food samples from the 2008 Canadian total diet study in Quebec City and dietary intake estimates. Food Addit Contam Part A Chem Anal Control Expo Risk Assess 2011; 28:791-798.

20. Seaborg E. EDCs: researchers and regulators argue the facts. Endocr News 2016; 18-22.

21. Soriano S, Alonso-Magdalena P, García-Arévalo M, Novials A, Muhammed SJ, Salehi A, Gustafsson JA, Quesada I, Nadal A. Rapid insulinotropic action of low doses of bisphenol-A on mouse and human islets of Langerhans: role of estrogen receptor b. PLoS ONE 2012; 7, e31109. doi:10.1371/journal. pone.0031109

22. D. Gentilcore D, Porreca I, Rizzo F, Ganbaatar E, Carchia E Mallardo M, De Felice M, Ambrosino C. Bisphenol A interferes with thyroid specific gene expression. Toxicology 2013; 304:21-31

23. Zhang YF, Ren XM, Li YY, Yao XF, Li CH, Qin ZF, Guo LH. Bisphenol A alternatives bisphenol $\mathrm{S}$ and bisphenol $\mathrm{F}$ interfere with thyroid hormone signaling pathway in vitro and in vivo. Environ Pollut 2017; pii: S0269-7491(17)33580-7. doi: 10.1016/j.envpol.2017.11.027.

24. Lee S, Kim C, Youn H, Choi K. Thyroid hormone disrupting potentials of bisphenol $\mathrm{A}$ and its analogues - in vitro comparison study employing rat pituitary (GH3) and thyroid follicular (FRTL-5) cells. Toxicol In Vitro 2017; 40:297-304. doi: 10.1016/j.tiv.2017.02.004

25. Sriphrapradang C, Chailurkit LO, Aekplakorn W, Ongphiphadhanakul B. Association between bisphenol A and abnormal free thyroxine level in men. Endocrine 2013; 44:441447. doi:10.1007/s12020-013-9889-y

26. Meeker JD, Ferguson KK. Relationship between urinary phthalate and bisphenol A concentrations and serum thyroid measures in U.S adults and adolescents from the National Health and Nutrition Examination Survey (NHANES) 2007-2008. Environ. Health Perspect 2011; 119:1396-1402 doi:10.1289/ehp.1103582.

27. Guignard D, Gayrard V, Lacroix MZ, Puel S, Picard-Hagen N, Viguié C. Evidence for bisphenol A-induced disruption of maternal thyroid homeostasis in the pregnant ewe at low level representative of human exposure. Chemosphere 2017; 182:458-467. Doi: 10.1016/j.chemosphere.2017.05.028

28. Aung MT, Johns LE, Ferguson KK, Mukherjee B, McElrath $\mathrm{TF}$, Meeker JD. Thyroid hormone parameters during pregnancy in relation to urinary bisphenol A concentrations: A repeated measures study. Environ Int 2017; 104:33-40. doi: 10.1016/ j.envint.2017.04.001.

29. Zhou Z, Zhang J, Jiang F, Xie Y, Zhang X, Jiang L. Higher urinary bisphenol A concentration and excessive iodine intake are associated with nodular goiter and papillary thyroid carcinoma Biosci Rep 2017 Jul 27;37(4). pii: BSR20170678. doi: 10.1042/ BSR20170678. 